\begin{tabular}{|c|c|c|}
\hline $\begin{array}{l}\text { ITC } 1 / 47 \\
\text { Journal ofInformation Technolo }\end{array}$ & \multicolumn{2}{|c|}{$\begin{array}{c}\text { Dimensionality Reduction Methods: The Comparison } \\
\text { of Speed and Accuracy }\end{array}$} \\
\hline $\begin{array}{l}\text { Vol. } 47 / \text { No. } 1 / 2018 \\
\text { pp. } 151-160\end{array}$ & Received 2017/08/11 & Accepted after revision 2018/01/25 \\
\hline $\begin{array}{l}\text { DOI 10.5755/j01.itc.47.1.18813 } \\
\text { @ Kaunas University of Technology }\end{array}$ & \multicolumn{2}{|c|}{ eross ${ }^{2}$ http://dx.doi.org/10.5r55/j01.itc.47.1.18813 } \\
\hline
\end{tabular}

\title{
Dimensionality Reduction Methods: The Comparison of Speed and Accuracy
}

\section{Jelena Zubova, Olga Kurasova}

Vilnius University, Institute of Mathematics and Informatics, Akademijos str. 4, LT-08663 Vilnius, Lithuania, e-mails: jelena.zubova@mii.vu.lt,olga.kurasova@mii.vu.lt

\section{Marius Liutvinavičius}

Vilnius University, Kaunas Faculty, Muitinès str. 8, LT-44280 Kaunas, e-mail: marius.liutvinavicius@khf.vu.lt

Corresponding author: jelena.zubova@mii.vu.lt

This research focuses on big data visualization that is based on dimensionality reduction methods. We propose a multi-level method for data clustering and visualization. It divides the whole data mining process into separate steps and applies particular dimensionality reduction method considering to analyzed data volume and type. The methods are selected according to their speed and accuracy. Therefore, we present a comparison of the selected methods according to these two criteria. Three groups of datasets containing different kind of data are used for methods evaluation. The factors that influence speed or accuracy are determined. The rank of investigated methods based on research results is presented in this paper.

KEYWORDS: big data, dimensionality reduction, data visualization.

\section{Introduction}

Big data analytics is the process of investigating big data to uncover hidden and useful information for better decisions. It involves visual presentation of data that enables to see hidden relations between objects which cannot be detected using conventional data analysis methods [14].

In this research, we focus on big data visualization that is based on dimensionality reduction methods. Our main goal is to find the most effective ways to analyse and visualize data of such type. Dimensionality reduction refers to the process of taking a data set with a usually large number of dimensions, and then creating a new data set with a fewer number of dimensions, that preserve as much of initial 
information as possible [6].

We propose a method which divides the data mining process into separate steps. At each stage, a particular dimensionality reduction and visualization method is applied considering to data volume and type. The methods are selected according to their speed and accuracy. Therefore, in the second section of this paper we present the comparison of selected dimensionality reduction methods.

When data are clustered and visualized, there is a possibility to see the parameters of each data group. The further analysis is performed only for the selected data cluster.

At the initial stage, the accuracy of method is not so important, so the fastest visualization method can be used. For the following dimensionality reduction steps, the demand for accuracy gradually increases. This requires using more accurate, but possibly slower methods. During each step, the selected data cluster is divided into smaller sets. At the end, the most accurate method processes the data. It would require too much resources at the beginning of dimensionality reduction, but at the end the data set is small enough to be processed in the most accurate way.

Most often in scientific literature there are just qualitative comparisons of different dimensionality reduction methods [2], [12], [11]. In some papers [6], [5], we can also find speed or accuracy comparisons of selected methods. The review of such researches leads to insight that some methods are faster, but less accurate and that other ones have opposite characteristics. However, there is a lack of general quantitative research of most popular methods that would compare both speed and accuracy.

Therefore, in this paper we investigate these wellknown methods: Multidimensional Scaling (MDS), Principal Component Analysis (PCA), Independent Component Analysis (ICA), Principal Curves, Locally Linear Embedding (LLE), and Isometric Mapping (Isomap).

\section{A Review of Dimensionality Reduction Methods}

A brief summary of the most popular dimensionality reduction methods is presented in this section. It is based on researches previously made by Fodor [2], Mizuta [7], Sorzano et. al. [12]. According to them, much of the data are highly redundant and can be efficiently brought down to a much smaller number of variables without a significant loss of information.

\section{Multidimensional scaling (MDS)}

Given $n$ items in a $d$-dimensional space and an $n x n$ matrix of proximity measures among the data items, MDS produces a $k$-dimensional, $k \leq d$, representation of the items such that the distances among the points in the new space reflect the proximities in the data [2]. In this research, we use $m d s O$ function from $\mathrm{R}$ package 'smacof' [11]. It solves the stress target function for symmetric dissimiliarities by means of the majorization approach (SMACOF) and reports the Stress-1 value (normalized).

\section{Figure 1}

A Multi-level method for big data visualisation

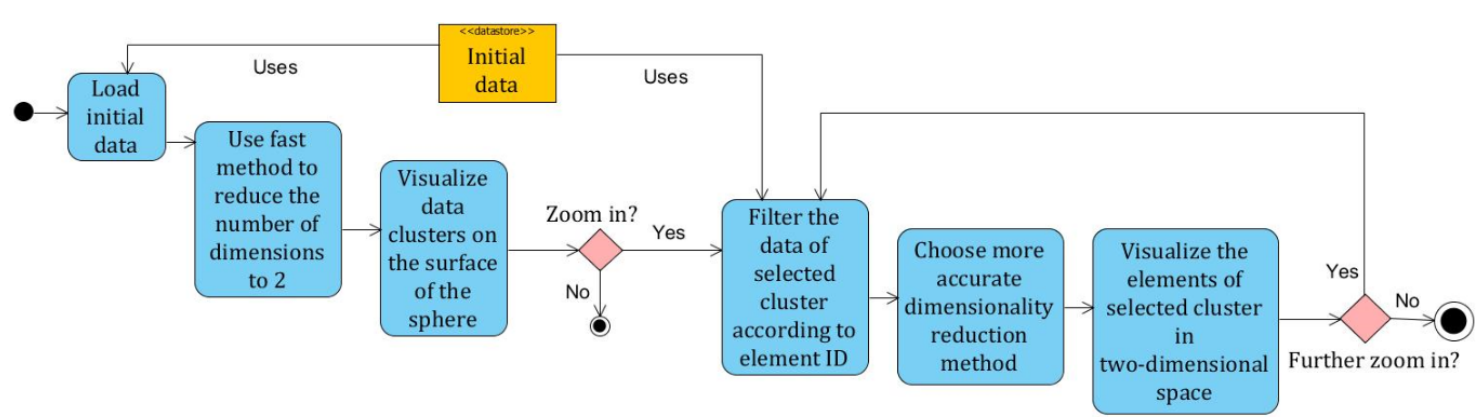


This function allows for fitting three basic types of MDS: ratio MDS (used in our case), interval MDS (polynomial transformation), ordinal MDS (also known as nonmetric MDS) [10].

\section{Principal components analysis (PCA)}

PCA is by far one of the most popular algorithms for dimensionality reduction [12]. It finds components that make projections uncorrelated by selecting the highest eigenvalues of the covariance matrix and maximizes retained variance [1]. PCA finds the principal components of the data, which correspond to the components along which there is the most variation [6].

\section{Independent component analysis (ICA)}

ICA is a higher-order method that seeks linear projections, not necessarily orthogonal to each other, that are as nearly statistically independent as possible. While PCA seeks uncorrelated variables, ICA seeks independent variables [2]. It should be noted that statistical independence is a much stronger condition than uncorrelatedness.

\section{Principal curves, surfaces and manifolds}

In situations where initial data have some curved structure methods like PCA do not work well. In such cases, approximating the curve by a straight line will not perform a good approximation of the original data. For such data type, the solution is to use principal curves, surfaces and manifolds [12]. Curve fitting to data is an important method for data analysis. When we obtain a fitting curve for data, the dimension of the data is nonlinearly reduced to one dimension [7].

\section{Locally linear embedding (LLE)}

LLE method is used to learn manifolds close to the data and project them onto them. For each item, it looks for the $K$-nearest neighbours and produces a set of weights for its approximation. This optimization is performed simultaneously for all items. Once the weights have been determined, it looks for points of lower dimension. The new points are reconstructed from its neighbours in the same way (with the same weights) as the items they represent [2].

\section{Isometric mapping (Isomap)}

If the distances between objects are measured as geodesic distances, then the MDS method is called Isomap. The geodesic distance between two points in a manifold is the one measured along the manifold itself; in practical terms, it is computed as the shortest path in a neighborhood graph connecting each observation to its $K$-nearest neighbors [2].

\section{Research Methodology}

The main goal of this research is to compare the speed and accuracy of the selected methods of visualization based on dimensionality reduction. $\mathrm{R}$ was chosen as a basis for analysis, because there are various open source packages that enable to execute and evaluate different dimensionality reduction methods. RStudio environment was used to perform the tasks.

\subsection{Data}

Three groups of different kinds of datasets were created for testing purposes.

\section{Randomly generated nonclustered data}

First of all, 50 different datasets containing randomly generated numbers were created with $\mathrm{R}$ function sample 0 . The number of columns is from 10 to 50 . The number of items is from 1000 to 10000 . Thus the smallest dataset is $1000 \times 10$ and the largest one is $10000 \times 50$.

\section{Randomly generated clustered data}

The second group contains 25 datasets of clustered data. The function genRandomClust from $\mathrm{R}$ package 'clusterGeneration' was used to generate cluster datasets with specified degree of separation [8]. Each dataset has four clusters. The number of columns is from 10 to 50. The number of items is from 1000 to 9 000 . The smallest dataset is $1000 \times 10$ and the largest one is $9000 \times 50$.

\section{Real financial data}

The third group contains 20 datasets of real financial data - stock ratios from finviz.com [13]. In total, there is information about 7000 companies. Each company is described by 50 parameters, which can be grouped into six categories: overview (price, volume, etc.), financial (ROI, ROA, etc.), valuation (EPS, P/E, etc.), performance (price changes, volatility, recommendations), technical (Beta, SMA, etc.), ownership.

The number of columns in datasets is from 10 to 50. The number of items is from 1000 to 7000 . The smallest dataset is $1000 \times 10$ and the largest one is 
$7000 \times 50$. In all cases of our research, the initial number of dimensions is reduced to two.

\subsection{The Evaluation Criteria}

We use two main criteria to compare different methods:

- Speed. It is measured as execution time of dimensionality reduction process.

- Accuracy. We use three different measures to evaluate the accuracy:

Stress - the measure got by solving the square loss function of MDS method. We used $\mathrm{R}$ function $m d s \mathrm{O}$ from package 'smacof' to find the stress value.

Spearman coefficient (The Spearman's Rank Correlation Coefficient). It is a statistical measure used to discover the strength of a link between two sets of data [3].

This ratio uses the ranks of variables instead of their values. Possible values range from -1 (strong negative relation) to 1 (strong positive relation). If ratio is equal to zero, this means there is no statistical link between datasets. To calculate this ratio, $\mathrm{R}$ function corO with method "spearman" was used.

Shannon entropy. We used R function entropy from package 'entropy' that estimates the Shannon entropy $\mathrm{H}$ of the random variable $\mathrm{Y}$ from the corresponding observed items [9], [4].

This estimator shows how accurate the projection got by using particular dimensionality reduction method retains the initial amount of information. A lesser value of this measure means better accuracy.

\section{Research Results}

The results of speed and accuracy comparison for each group of data are presented in this section. At the end, the overall comparison is made.

\subsection{Randomly Generated Nonclustered Data}

In the first case, randomly generated nonclustered datasets are used for investigation.

\section{The speed of methods}

As results show, MDS (smacof), Isomap and LLE methods have the same characteristics:

- When the number of instances increases, the execution time also increases.
- The initial amount of dimensions does not have significant effect on the time of execution.

Fig. 2 shows the execution time of MDS (smacof) method for datasets that contain ten columns, but differ in number of rows. The charts of execution time for the datasets having more columns look the same, because this factor has no influence. However, Isomap is much slower (this can be seen in Fig. 6).

\section{Figure 2}

Execution time of MDS (smacof) method

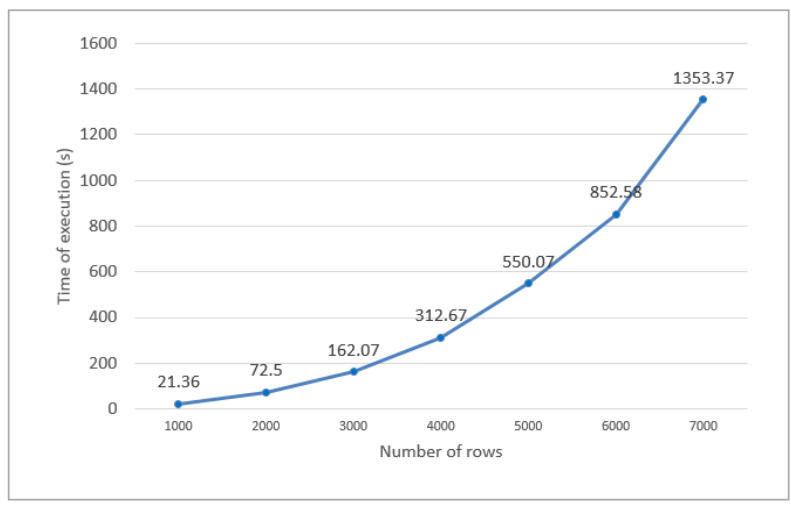

For PCA, the execution time increases just slightly in both cases: when the number of rows increases and when the number of columns increases (Fig. 3).

\section{Figure 3}

Execution time of PCA

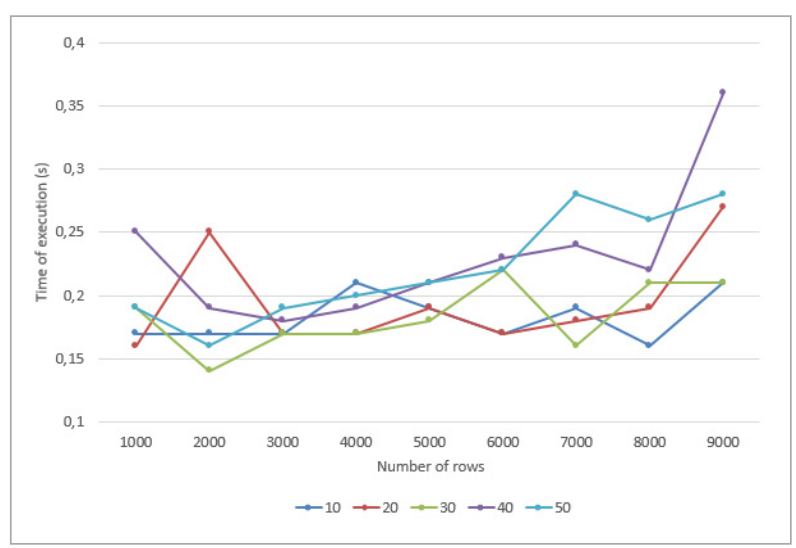

The execution time of ICA is similar to PCA. Only Principal curves distinguish by regular increase of execution time in both cases (when the number of dimensions and items increase) (Fig. 4). 
Figure 4

Execution time of Principal curves

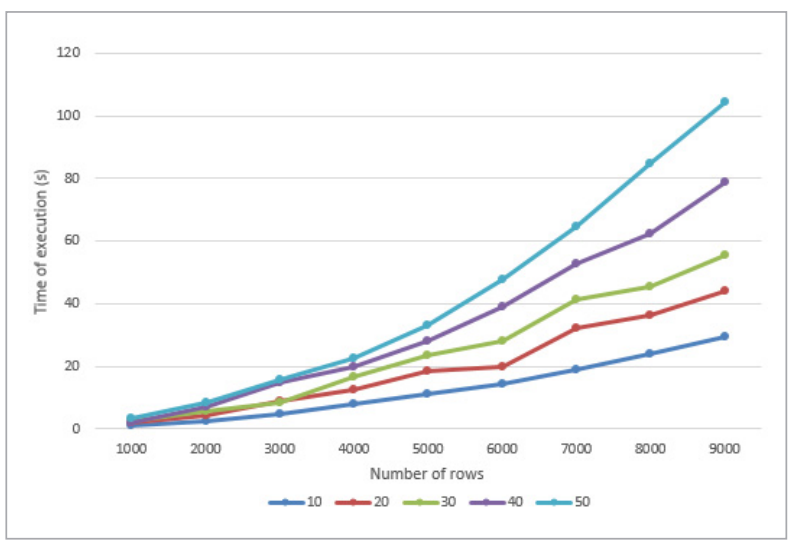

In Fig. 6, the execution times of tested methods are compared. The number of items is from 1000 to 10 000. The initial number of dimensions does not have significant influence for any method, so only one case with 40 dimensions is presented.

It should be noted that LLE could not process the datasets with more than 9000 rows, Isomap could not
Figure 5

A Comparison of execution times

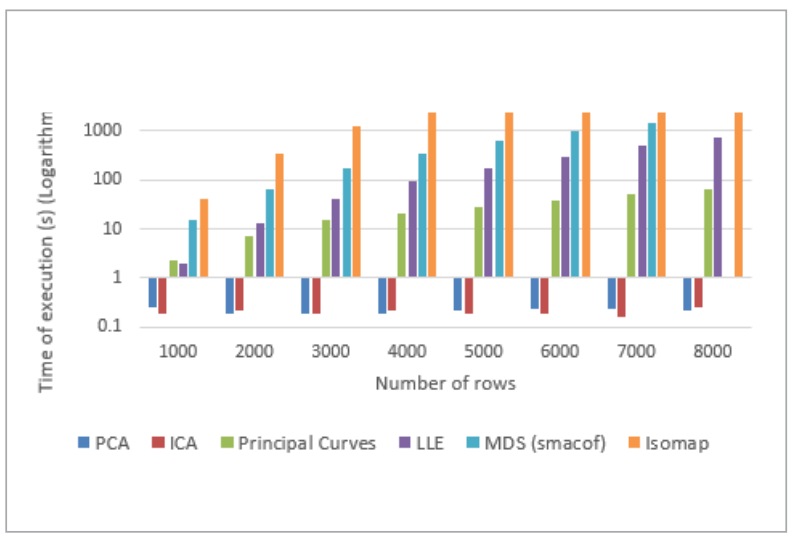

process more than 8000 rows and for MDS (smacof) the limit was 7000 rows. This was due to the lack of RAM. In Fig. 5, the speed of methods is presented in a logarithmic scale. PCA and ICA are the fastest. Principal curves, MDS and LLE are much slower. However, Isomap is the slowest (its execution time is significantly longer than that of others).

\section{Figure 6}

A Comparison of execution times

\begin{tabular}{|c|c|c|c|c|c|c|c|c|c|c|}
\hline & 1000 & 2000 & 3000 & 4000 & 5000 & 6000 & 7000 & 8000 & 9000 & 10000 \\
\hline PCA & 0.25 & 0.19 & 0.18 & 0.19 & 0.21 & 0.23 & 0.24 & 0.22 & 0.36 & 0.28 \\
\hline ICA & 0.18 & 0.22 & 0.19 & 0.22 & 0.18 & 0.18 & 0.16 & 0.25 & 0.29 & 0.23 \\
\hline Principal Curves & 2.21 & 6.89 & 14.73 & 19.98 & 28.29 & 39.22 & 52.55 & 62.47 & 78.98 & 105.88 \\
\hline LLE & 2.00 & 13.22 & 41.51 & 93.66 & 178.67 & 304.12 & 486.33 & 727.57 & 1029.79 & - \\
\hline MDS (smacof) & 15.76 & 65.64 & 173.64 & 340.48 & 624.07 & 981.79 & 1482.42 & & - & - \\
\hline Isomap & 39.75 & 350.41 & 1229.72 & 3168 & 6222.66 & 11078.34 & 17710.56 & 41077.29 & - & \\
\hline
\end{tabular}

\section{The accuracy of methods}

For all the investigated methods, we found that the same rules apply:

_ When the number of items increases, the accuracy does not change.

_ When the number of initial dimensions increases, it leads to worse accuracy.

This was confirmed by all measures. However, the level of accuracy reduction is not the same for different methods. Figures 7 and 8 compare the accuracy of all analysed methods. As the number of instances does not make significant influence, we show only the cases with 7000 items.
Figure 7

A Comparison of the accuracy measures

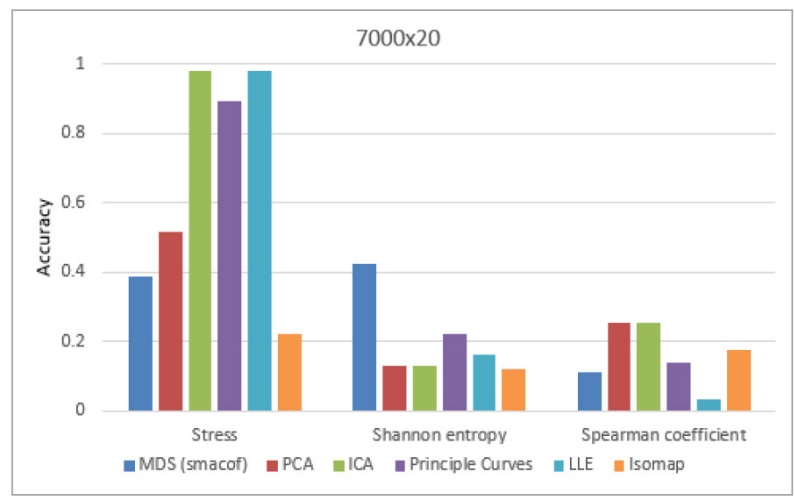


Figure 8

A Comparison of the accuracy measures

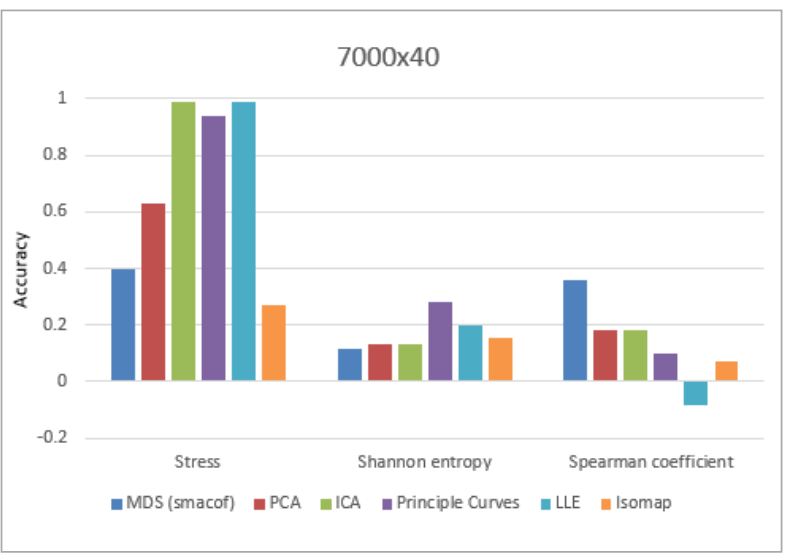

In all cases with different number of initial dimensions, the results are similar. Therefore, we present only two of them: 20 dimensions and 40 dimensions.

The results show that PCA and MDS were the most accurate with our datasets. LLE showed the worst accuracy.

\section{The rank of methods}

Fig. 9 summarizes the results of nonclustered data case. We ranked all investigated methods by their speed and accuracy (" 6 " means the highest score and "1" stands for the worst score).

PCA and ICA are the fastest methods. MDS is the most accurate, but slower. Principal curves showed moderate results. The results of LLE and Isomap are the worst. Even though Isomap is significantly slower, its accuracy in some cases can be the best.

\section{Figure 9}

A Comparison of methods by speed and accuracy

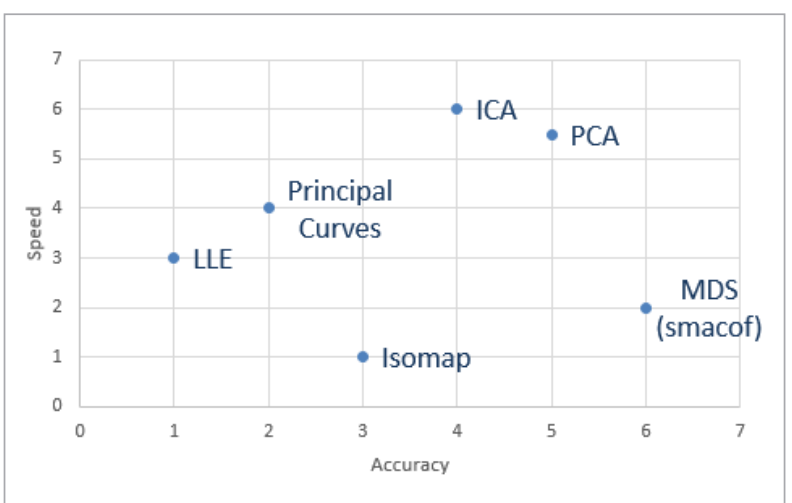

\subsection{Randomly generated clustered data}

In the second case, randomly generated clustered datasets are used for the investigation.

\section{The speed of methods}

For MDS (smacof), Isomap and LLE, the same trends as with nonclustered data can be seen:

- When the number of items increases, the execution time also increases.

- The initial amount of dimensions does not have significant effect on the time of execution.

Fig. 10 shows the execution times of these methods for datasets from $1000 \times 10$ to $7000 \times 10$.

\section{Figure 10}

Execution time of MDS (smacof), Isomap and LLE methods

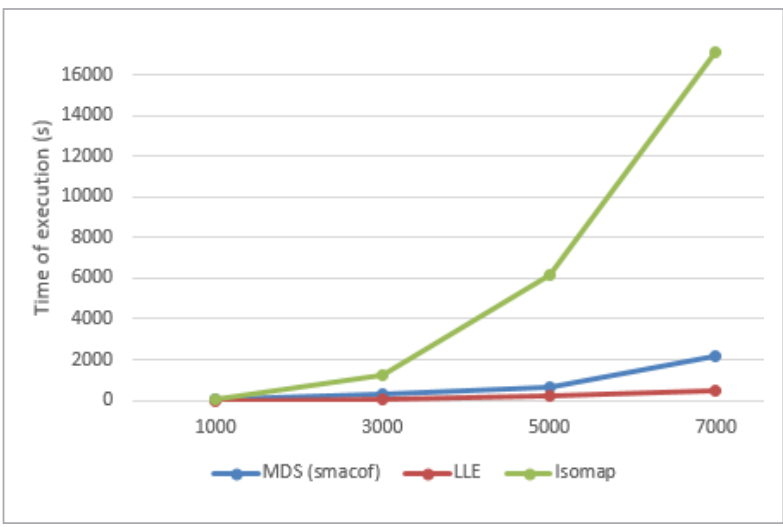

For PCA, the execution time slightly increases (with some exceptions) when both the number of rows and the number of columns increases. In this case, the execution time of ICA is also similar to PCA. With clustered data, there is no such obvious regular increase of the execution time when using Principal curves method (Fig. 11), which can be seen in Fig. 4.

In Fig. 12, the execution times of different methods are compared. The number of items is $1000,3000,5000$, 7000 and 9000 . In this case, the number of dimensions does not have significant influence for any method, so only one case with 40 dimensions is presented.

The results are similar to those that were obtained previously with nonclustered data (Fig. 5). However, in this case, LLE, MDS (smacof) and Isomap could not process the datasets with 9000 rows. 
Figure 11

Execution time of Principle curves method

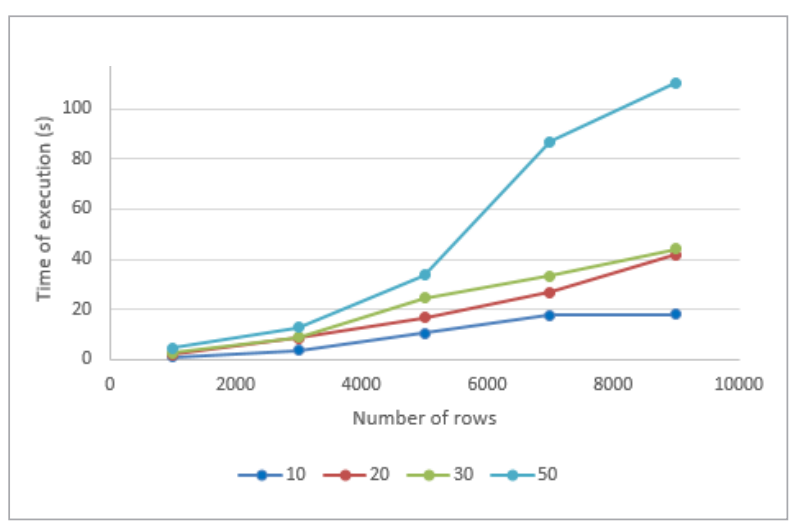

Figure 12

A Comparison of execution times (with 40 initial dimensions)

\begin{tabular}{|l|r|r|r|r|r|}
\hline & $\mathbf{1 0 0 0}$ & $\mathbf{3 0 0 0}$ & $\mathbf{5 0 0 0}$ & $\mathbf{7 0 0 0}$ & $\mathbf{9 0 0 0}$ \\
\hline PCA & 0.21 & 0.2 & 0.21 & 0.25 & 0.23 \\
\hline ICA & 0.19 & 0.17 & 0.19 & 0.19 & 0.17 \\
\hline Principal Curves & 2.62 & 11.91 & 22.81 & 49.08 & 109.98 \\
\hline LLE & 2.14 & 41.45 & 184.04 & 484.42 & - \\
\hline MDS (smacof) & 14.81 & 185.36 & 42.125 & 1750.67 & - \\
\hline Isomap & 38.33 & 1219.35 & 6297.34 & 18008.4 & -
\end{tabular}

\section{The accuracy of methods}

For all methods, we found that the same rules apply as with nonclustered data:

1 When the number of instances increases, the accuracy does not change.

2 When the number of initial dimensions increases, it leads to worse accuracy.

\section{Figure 13}

A Comparison of accuracy ratios (dataset $7000 \times 40$ )

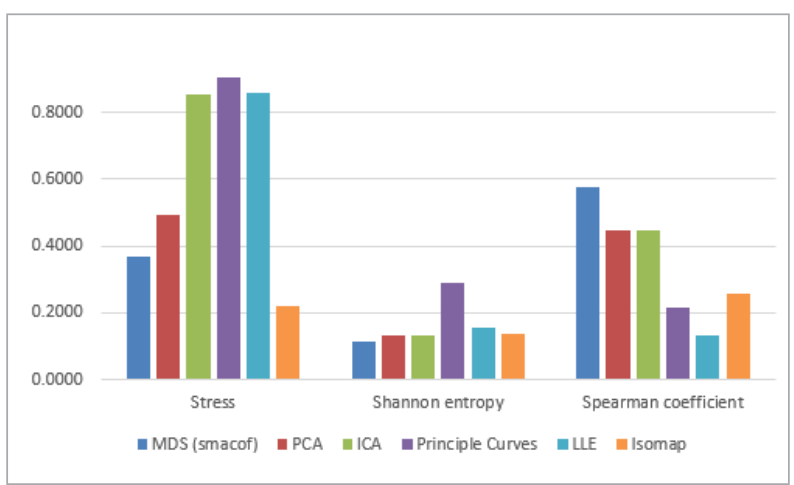

Fig. 13 shows the accuracy values got with dataset that contains 7000 rows and 40 columns. MDS (smacof) is the most accurate by two measures: Shannon entropy and Spearman coefficient. However, according to Stress, Isomap is more accurate than MDS (smacof). PCA and ICA showed the moderate results. The accuracy of LLE and Principle curves is the worst.

The results of speed and accuracy with clustered data are almost the same as with nonclustered data.

\subsection{Real Financial Data}

In the third case, the real stock data are used for comparison of dimensionality reduction methods.

\section{The speed of methods}

It may seem that MDS (smacof) has the same characteristics (when the number of instances increases the execution time also increases; the initial amount of dimensions does not have significant effect on the time of execution). However, in the case with real data, we found that execution time slightly increases when the number of initial dimensions increases (Fig. 14). This contrary relationship is unusual and needs further investigation.

\section{Figure 14}

Execution time of MDS (smacof) method

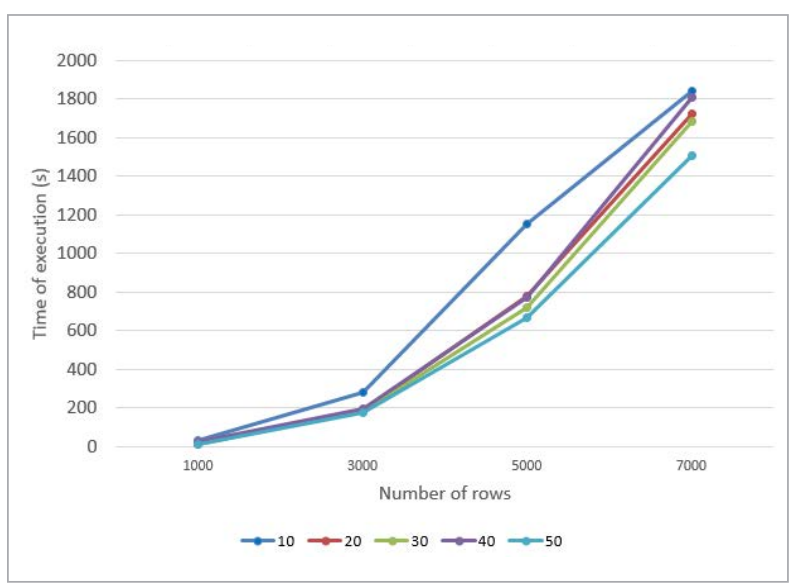

For the remaining methods, the trends of speed are the same as in previous cases. However, it was impossible to process the real data with LLE method. It found data too much correlated. 
Figure 15

A Comparison of execution times

\begin{tabular}{l|r|r|r|r} 
& $\mathbf{1 0 0 0}$ & $\mathbf{3 0 0 0}$ & $\mathbf{5 0 0 0}$ & $\mathbf{7 0 0 0}$ \\
\hline PCA & 0.18 & 0.15 & 0.19 & 0.23 \\
\hline ICA & 0.19 & 0.17 & 0.16 & 0.20 \\
\hline Principal Curves & 4.43 & 17.27 & 38.89 & 81.25 \\
\hline MDS (smacof) & 22.58 & 198.82 & 774.7 & 1809.37 \\
\hline Isomap & 42.37 & 1130.76 & 6175.44 & 16599.5 \\
\hline LLE & - & - & - & - \\
\hline
\end{tabular}

\section{The accuracy of methods}

With real data, we could not get the measures not only for LLE method, but also the Stress value of ICA. This confirms that all methods can cope with generated data, but real world situations may cause issues to them.

Fig. 16 shows the results in case with 7000 items and 40 dimensions. MDS (smacof), PCA, ICA and Isomap show similar results with all datasets (accuracy depends on the initial amount of dimensions, but the trends remain the same).

However, with Principle curves we could not confirm one rule for all datasets. Fig. 17 shows that when the number of initial dimensions constantly increases, the values of Spearman coefficient and Shannon entropy fluctuates. This leads to suggestion that information, which can be extracted from data, has impact on the accuracy of dimensionality reduction.

This is why adding more columns of randomly generated data is not the same as adding more real data, which can add completely different aspects for analyzed subject.

Fig. 18 shows that more items lead to better accuracy. This feature is seen only with real data.

\section{Figure 16}

A Comparison of accuracy (dataset $7000 \times 40$ )

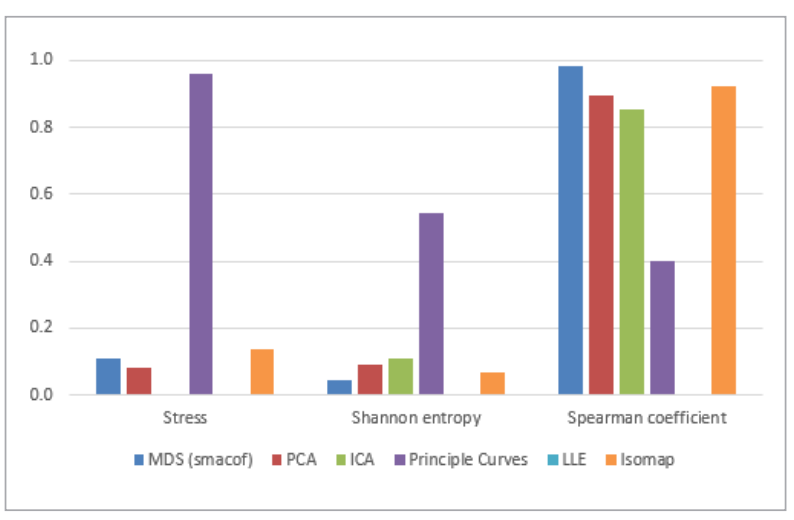

Figure 17

The accuracy of Principal curves

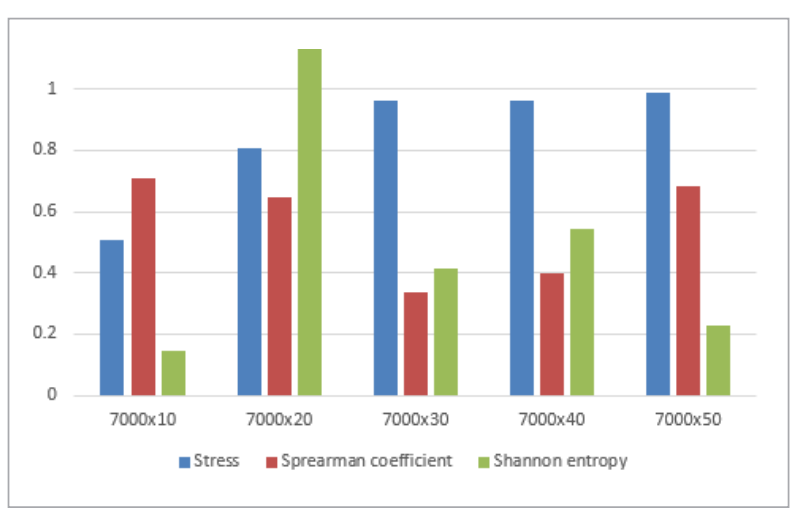

Figure 18

The accuracy of Principal curves

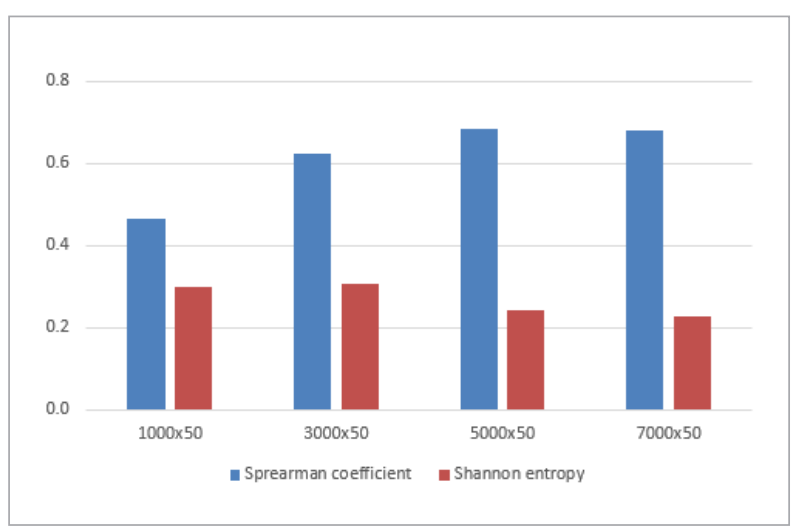

\section{The rank of methods}

Fig. 19 shows the rank of methods according to their speed and accuracy while processing the real data.

\section{Figure 19}

A Comparison of speed and accuracy

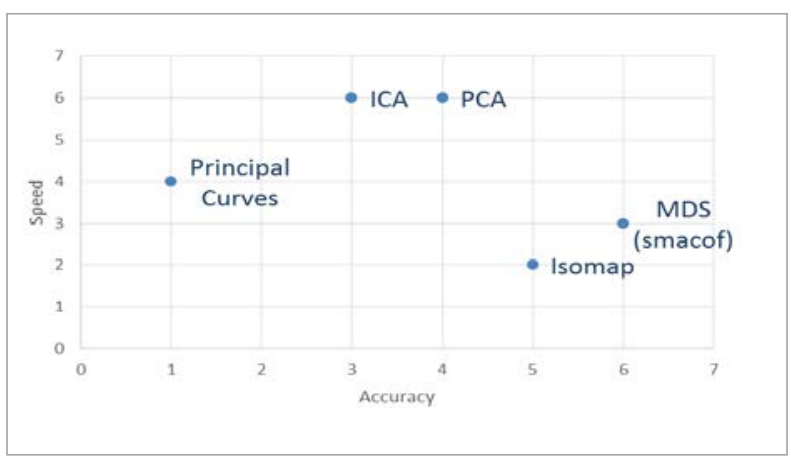


The results show that MDS is the most accurate method. However, it is not as fast as PCA or ICA. The latter two are the fastest methods, but they showed moderate accuracy values. The speed of ICA is the same as PCA, but it is not so accurate.

\subsection{The Overall Comparison}

In this section, we present how the speed and accuracy of dimensionality reduction methods depend on the kind of data. Fig. 20 shows that the kind of data is not important for the speed of methods. It does not affect the time of execution.

However, it has infuence on the accuracy. Clustered data have better Stress values than nonclustered data. Moreover, PCA, MDS (smacof) and Isomap showed best accuracy exactly with real stock data (Fig. 21).

According to Spearman coefficient (Fig. 22), the best accuracy is also achieved when processing the real

\section{Figure 20}

Execution times for different kind of data

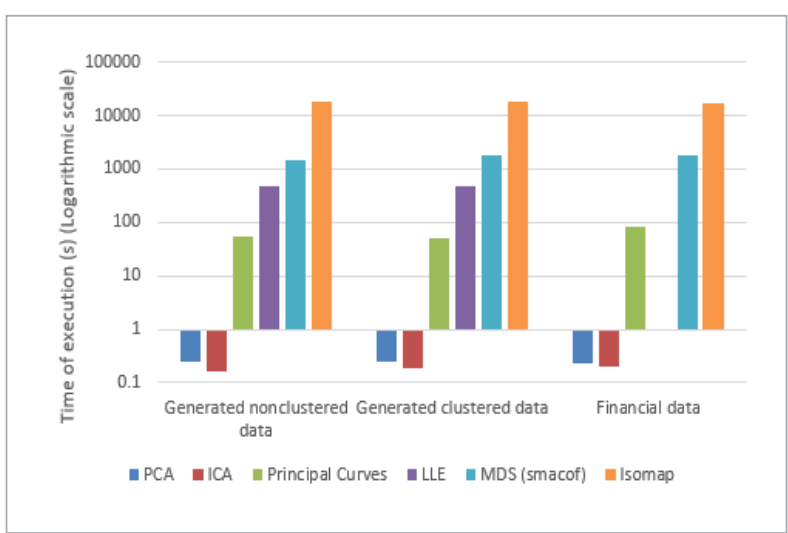

\section{Figure 21}

A Comparison of accuracy (Stress)

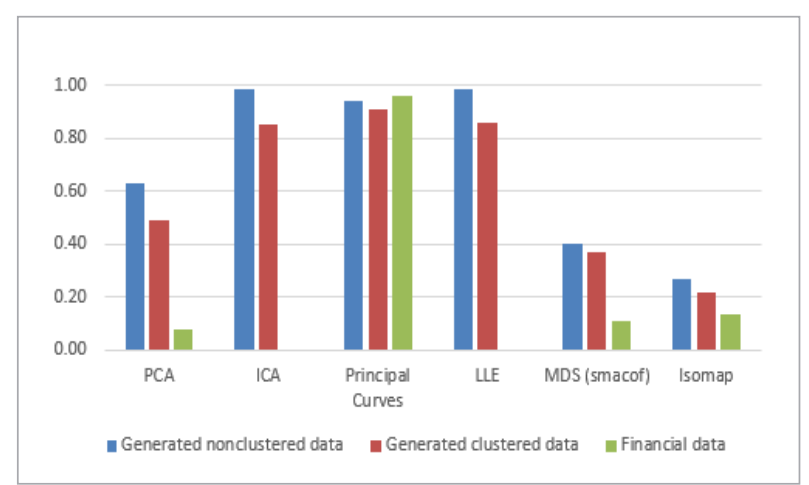

Figure 22

Accuracy measures: Spearman coefficient

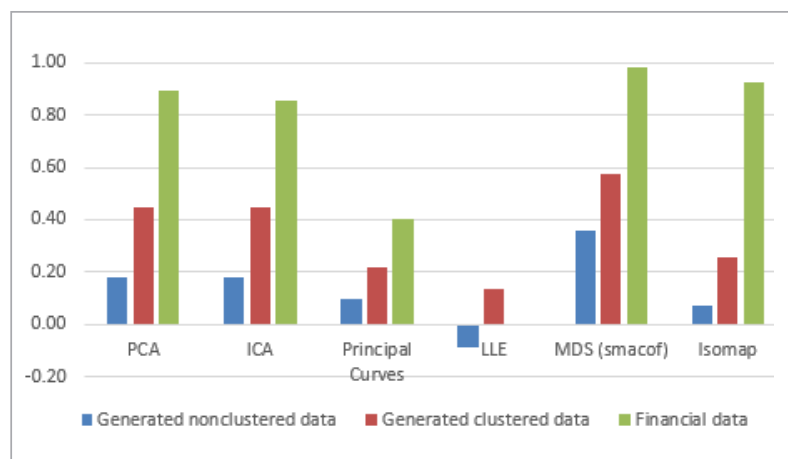

Figure 23

Accuracy measures: Shannon entropy

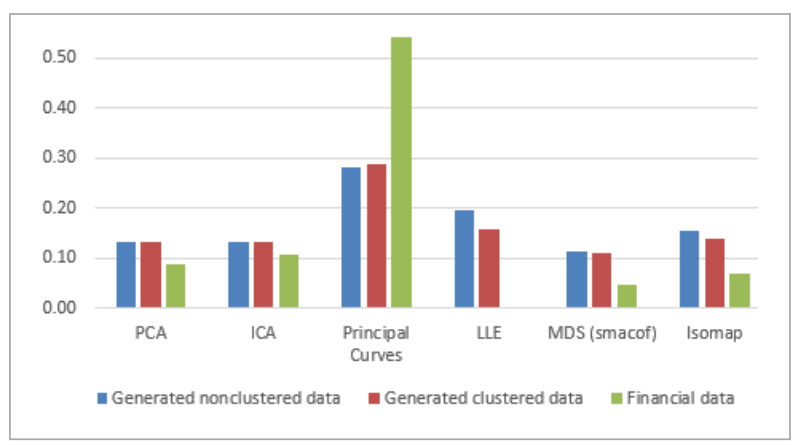

data. Clustered data also have higher accuracy values than nonclustered data.

According to Shannon entropy (Fig. 23), there is no significant difference of accuracy between clustered and nonclustered data. However, again, the accuracy is much better in case with the real data (except Principal curves method).

\section{Conclusions}

In this paper, we presented the methodology that divides data visualisation process into separate steps. For each step, individual dimensionality reduction and visualization method is applied considering to data volume and type. The particular methods are selected according to their speed and accuracy. Therefore, we presented the comparison of dimensionality reduction methods according to these two criteria. Three different measures were used to evaluate the accuracy: 
Stress, Spearman coefficient and Shannon entropy. All methods were tested with three groups of different kind of data: nonclustered randomly generated data, clustered randomly generated data and real financial data.

Several rules were confirmed for randomly generated data (both clustered and nonclustered). When the number of items increases, the execution time also increases. However, the initial amount of dimensions does not have a significant effect on the time of execution. For accuracy, the situation is the opposite. When the number of items increases, the accuracy does not change, but when the number of initial dimensions increases, it leads to worse accuracy.

Meanwhile, in the case with real data, we found that execution time can slightly increase when the number of initial dimensions increases. It was also impos-

\section{References}

1. Domeniconi, C. Comparison of Principal Component Analysis and Random Projection in Text Mining. INFS, 2004, 795 .

2. Fodor, I. K. A Survey of Dimension Reduction Techniques. Center for Applied Scientific Computing, Lawrence Livermore National Laboratory, 2002.

3. Hauke, J., Kossowski, J. Comparison of Values of Pearson's and Spearman's Correlation Coefficients on the Same Sets of Data. Quaestiones Geographicae, 2011, 30(2), 87-93.

4. Hausser, J., Strimmer, K. Entropy Inference and the James-Stein Estimator, with Application to Nonlinear Gene Association Networks. Journal of Machine Learning Research, 2009, 10, 1469-1484.

5. Kim, H., Howland, P., Park, H. Dimension Reduction in Text Classification with Support Vector Machines. Journal of Machine Learning Research, 2005, 6, 37-53.

6. Menon, A. K. Random Projections and Applications to Dimensionality Reduction. School of Information Technologies, The University of Sydney. http://citeseerx.ist.psu.edu/viewdoc/download?doi=10.1.1.164.640\&rep=rep1\&type=pdf (last accessed 2 November 2017).

7. Mizuta, M. Dimension Reduction Methods. Humboldt-Universität Berlin, Center for Applied Statistics and Economics (CASE), 2007, 15. sible to process the real data with LLE method and get Stress values of ICA. This shows that real world situations may cause issues to particular methods. The results also show that more instances of real data lead to better accuracy. They also show that the kind of data is not important for the speed of methods, but it has influence on the accuracy. Clustered data have better values of accuracy metrics than nonclustered data. The best accuracy is achieved when processing the real data.

The results show that MDS is the most accurate method, but not as fast as PCA or ICA. These are the fastest methods, but they showed moderate accuracy values. Principal curves and LLE showed the worst results. Isomap was significantly slower, but its accuracy in some cases can be the best.

8. R Package 'clusterGeneration' - Random Cluster Generation (with Specified Degree of Separation), 2015. https://cran.r-project.org/web/packages/clusterGeneration/clusterGeneration.pdf (last accessed 2 November 2017).

9. R Package 'entropy' - Estimation of Entropy, Mutual Information and Related Quantities. https://cran.r-project.org/web/packages/entropy/entropy.pdf (last accessed 2 November 2017).

10. R Package 'smacof' - Multidimensional Scaling, 2017. https://cran.r-project.org/web/packages/smacof/smacof.pdf (last accessed 2 November 2017).

11. Rosaria, R. S., Adae, I., Hart, A., Berthold, M. Seven Techniques for Dimensionality Reduction. Knime, 2014.https:// www.knime.com/blog/seven-techniques-for-data-dimensionality-reduction (last accessed 2 November 2017).

12. Sorzano, C. O. S., Vargas, J., Montano, A. P. A Survey of Dimensionality Reduction Techniques. 2014. https:// arxiv.org/abs/1403.2877 (last accessed 2 November 2017).

13. Stock Ratios. http://finviz.com/ (last accessed 2 November 2017)

14. Zubova, J., Kurasova, O., Liutvinavicius, M. Parallel Computing for Dimensionality Reduction. Information and Software Technologies, Springer-Verlag, 230-241, 2016. ISBN 978-3-319-46254-7. 